\title{
Discrepancy between MRA and DSA in identifying the shape of small intracranial aneurysms
}

\author{
Youngseok Kwak, MD, ${ }^{1}$ Wonsoo Son, MD, ${ }^{2}$ Yong-Sun Kim, MD, PhD, ${ }^{3}$ Jaechan Park, MD, PhD, ${ }^{2}$ and \\ Dong-Hun Kang, MD²
}

1Department of Neurosurgery, School of Medicine, Catholic University of Daegu; and Departments of 2 Neurosurgery and ${ }^{3}$ Radiology, School of Medicine, Kyungpook National University, Daegu, Republic of Korea

\begin{abstract}
OBJECTIVE The authors evaluated the sensitivity and accuracy of MRA in identifying the shape of small-sized unruptured intracranial aneurysms.

METHODS Small (<7 mm) unruptured intracranial aneurysms initially detected by MRA and confirmed by DSA between January 2017 and December 2018 were morphologically reviewed by neuroradiologists. Regularity or irregularity of aneurysm shape was analyzed by two independent reviewers using MRA without DSA results. DSA findings served as the reference standard for aneurysm shape. Irregular shape, which in small aneurysms is associated with a higher likelihood of rupture, was defined as positive, and MRA sensitivity, specificity, and accuracy were determined by using evaluations based on location, size, and MRA magnetic strength (1.5T vs 3T MRA). Multivariate analysis was performed to determine risk factors for false-negative MRA results for irregularly shaped aneurysms.

RESULTS In total, 652 unruptured intracranial aneurysms in 530 patients were reviewed for this study. For detecting aneurysm shape irregularity, the overall MRA sensitivity was $60.4 \%$ for reviewer 1 and $60.9 \%$ for reviewer 2 . Anterior cerebral artery aneurysms had the lowest sensitivity for location (36.7\% for reviewer $1,46.9 \%$ for reviewer 2$)$; aneurysms sized $<3 \mathrm{~mm}$ had the lowest sensitivity for size (26.7\% for both reviewers); and $1.5 T$ MRA had lower sensitivity and accuracy than 3T MRA. In multivariate analysis, location, size, and magnetic strength of MRA were independent risk factors for false-negative MRA results for irregularly shaped aneurysms.
\end{abstract}

CONCLUSIONS MRA had a low sensitivity for detecting the irregular shape of small intracranial aneurysms. In particular, anterior cerebral artery location, aneurysm size $<3 \mathrm{~mm}$, and detection with 1.5T MRA were associated with a higher risk of irregularly shaped aneurysms being misjudged as regular.

https://thejns.org/doi/abs/10.3171/2020.4.JNS20128

KEYWORDS magnetic resonance angiography; intracranial aneurysm; aneurysm shape; digital subtraction angiography; sensitivity; accuracy; vascular disorders

$\mathrm{A}$ LTHOUGH digital subtraction angiography (DSA) is regarded as the standard imaging modality for diagnosing intracranial aneurysms, magnetic resonance angiography (MRA) has been recently and widely used for screening of intracranial aneurysms. MRA is free of radiation and less invasive, less expensive, and less time consuming than DSA. Several studies have reported a high accuracy for detecting aneurysms with MRA. However, there have been only a few reported studies of the use of MRA to determine aneurysm shape. Moreover, published reports on MRA for irregular aneurysm shape have shown mixed or inconclusive results, and most of these studies had only a small cohort of study patients. ${ }^{1-4}$
Large-sized aneurysms ( $\geq 7 \mathrm{~mm}$ ) have a high risk of rupture and tend to be treated regardless of shape. However, when the aneurysm size is small there is a higher risk of rupture if the shape is irregular (with a small bleb or lobulation). ${ }^{5,6}$ Therefore, accurate identification of shape for small-sized aneurysms is essential for therapeutic decision making. However, there has been a lack of studies that focus on identifying shape with MRA for small-sized aneurysms. Further, we occasionally encounter cases in practice in which the shape of the aneurysm determined with MRA differs from that determined with DSA (Fig. 1). Therefore, in the present study, we aimed to evaluate the accuracy of MRA for identifying the shape of small-sized

ABBREVIATIONS ACA = anterior cerebral artery; $A U C=$ area under the ROC curve; ICA = internal carotid artery; $M C A=$ middle cerebral artery; $N P V=$ negative predictive value; $P P V=$ positive predictive value; $R O C=$ receiver operating characteristic.

SUBMITTED January 13, 2020. ACCEPTED April 24, 2020.

INCLUDE WHEN CITING Published online July 24, 2020; DOI: 10.3171/2020.4.JNS20128. 

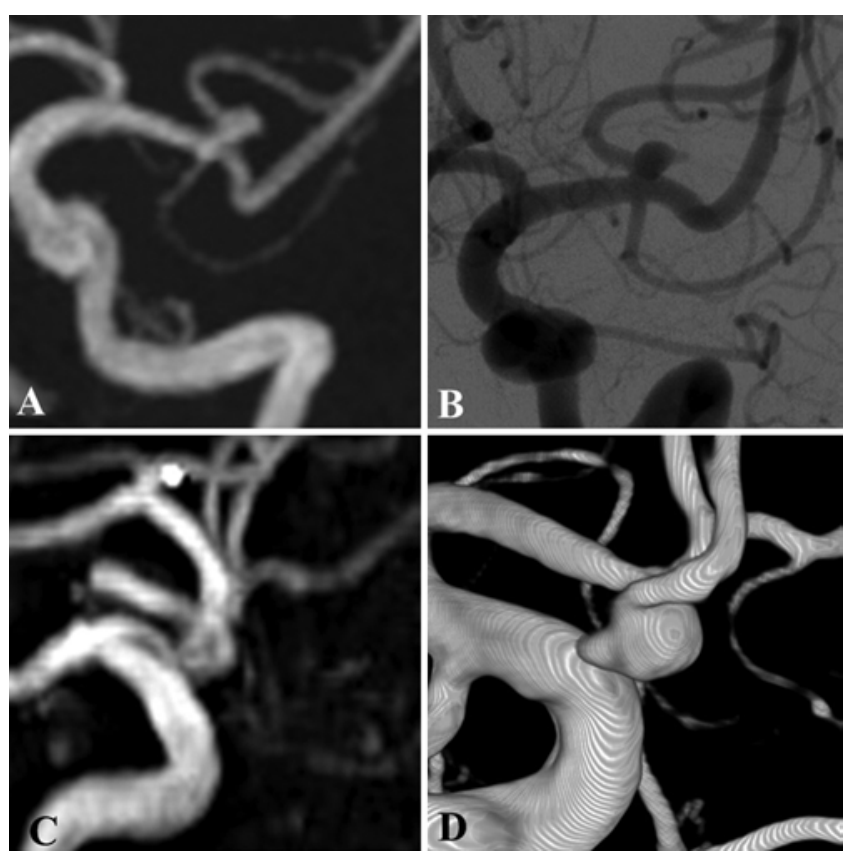

FIG. 1. A: MRA showing an MCA aneurysm with a regular shape. B: A small bleb of the aneurysm can definitely be seen on DSA, which cannot be seen on MRA. C: An anterior communicating artery aneurysm is partially obscured by the ICA on MRA. D: In the DSA, a small bleb is identified in an overlapped area on the MRA.

unruptured aneurysms. To our knowledge, this is the first study to evaluate MRA specifically regarding the shape of small intracranial aneurysms.

\section{Methods}

\section{Patient and Aneurysm Selection}

We reviewed our DSA database for patients with unruptured intracranial aneurysms that were initially diagnosed or suspected based on MRA performed between January 2017 and December 2018. For multiple aneurysms in a single patient, each aneurysm was evaluated separately. We excluded patients or aneurysms using the following criteria: 1) aneurysm with a maximum diameter $\geq 7 \mathrm{~mm}$, 2) traumatic or dissecting aneurysm, 3) recurrent aneurysm after coiling or clipping, 4) interval between MRA and DSA examination $>2$ months, and 5) aneurysms suspected on MRA but ruled out in DSA (e.g., junctional dilatation, normal anterior communicating artery).

\section{Image Acquisition}

We included only high-field (1.5T or 3T) time-of-flight MRA. A small number of patients had both time-of-flight MRA and gadolinium-enhanced MRA. We excluded gadolinium-enhanced MRA images from this study. Since patients had MRA performed at different facilities, the MR scanners and MRA protocols used were often different from patient to patient. Twenty-one models of MR equipment from 5 companies (Canon Medical Systems, GE Healthcare, Hitachi Medical Systems, Philips Healthcare, and Siemens Healthineers) were included in this study. However, the imaging procedures used standard algorithms and were of satisfactory image quality. We excluded a small minority of MRA images that had a low- to midfield (below 1.5T) MRA or poor visualization due to artifacts.

DSA was performed with a biplane angiography system (Allura, Philips Healthcare). We acquired anteroposterior and lateral images from 2D DSA for the bilateral internal carotid artery (ICA) and vertebral artery and then performed rotational angiography on affected or suspected arteries. We added 2D DSA of the profile angle, which was obtained through $3 \mathrm{D}$ reconstruction with rotational angiography, to identify the shape of the aneurysm. We excluded patients who did not undergo rotational angiography.

\section{Image Analysis}

The source images and maximum-intensity projection images of MRA were reviewed by two neuroradiologists (Y.K. and D.H.K.). A small number of patients in our study had undergone volume-rendering MRA, but those images were excluded from evaluation, as volume rendering could affect other variables.

The reviewers independently analyzed aneurysm morphology with MRA without DSA results. DSA findings, the reference standard regarding aneurysm shape, were then decided by consensus between two reviewers. The maximum aneurysm diameter (in millimeters) was measured through $3 \mathrm{D}$ reconstruction images from rotational angiography.

Aneurysm morphology was categorized as follows: 1, regular; 2, uncertain; or 3 irregular. Regular and irregular shapes were defined according to the study of Lindgren et al., ${ }^{6}$ who suggested that irregularly shaped aneurysms had a risk of rupture, irrespective of size. "Regular" was defined as "the aneurysm surface was smooth and regular in all projections," and "irregular" was defined as "small bleb(s) or secondary aneurysm(s) were protruding from the aneurysm fundus in any images, or when the aneurysm fundus was clearly bi- or multilobular.'6

\section{Statistical Analysis}

The categorical, demographic, and basic characteristic variables, expressed as numbers and percentages, were compared using the chi-square test. Continuous variables were expressed as the mean $\pm \mathrm{SD}$ and compared with an unpaired t-test if normally distributed. Irregular shape was defined as positive, and the diagnostic performance parameters of MRA compared with DSA were expressed in terms of sensitivity, specificity, positive predictive value (PPV), negative predictive value (NPV), accuracy, and area under the receiver operating characteristic (ROC) curve (AUC). We investigated whether MRA varies in detecting irregular shape relative to location (anterior cerebral artery [ACA], middle cerebral artery [MCA], cavernousclinoid-ophthalmic segment of the internal carotid artery [ICA], and communicating-segment bifurcations of the ICA, vertebral, and basilar arteries) and size $(<3,3-4.9$, and 5-6.9 $\mathrm{mm}$ ) of the aneurysm, and compared irregular shape detection with $1.5 \mathrm{~T}$ versus 3T MRA. The chi-square test was used to compare proportion values for sensitivity, 
TABLE 1. Baseline characteristics of patients and aneurysms

\begin{tabular}{|c|c|c|c|c|}
\hline & \multicolumn{3}{|c|}{ Aneurysms } & \multirow{2}{*}{$\begin{array}{c}p \\
\text { Value }\end{array}$} \\
\hline & All & Regular Shape & Irregular Shape & \\
\hline Patient age, yrs & $60.45 \pm 10.43$ & $60.24 \pm 10.45$ & $60.93 \pm 10.40$ & 0.428 \\
\hline Patient sex, n & & & & 0.229 \\
\hline Male & 161 & 105 & 56 & \\
\hline Female & 491 & 345 & 146 & \\
\hline Location & & & & $<0.001$ \\
\hline ACA & $111(17.0)$ & $62(13.8)$ & $49(24.3)$ & \\
\hline MCA & $168(25.8)$ & $98(21.8)$ & $70(34.7)$ & \\
\hline ICA cav-cli-oph & $217(33.3)$ & $181(40.2)$ & $36(17.8)$ & \\
\hline ICA com-bif & $113(17.3)$ & $73(16.2)$ & $40(19.8)$ & \\
\hline VBA & $43(6.6)$ & $36(8.0)$ & $7(3.5)$ & \\
\hline Size, mm & $3.70 \pm 1.45$ & $3.28 \pm 1.34$ & $4.66 \pm 1.22$ & $<0.001$ \\
\hline Size group & & & & $<0.001$ \\
\hline$<3 \mathrm{~mm}$ & $235(36.0)$ & $220(48.9)$ & $15(7.4)$ & \\
\hline $3-4.9 \mathrm{~mm}$ & 268 (41.1) & $169(97.6)$ & $99(49.0)$ & \\
\hline $5-6.9 \mathrm{~mm}$ & $149(22.9)$ & $61(13.6)$ & $88(43.6)$ & \\
\hline MRA field strength & & & & 0.904 \\
\hline $1.5 \mathrm{~T}$ & $454(69.6)$ & $314(69.8)$ & $140(69.3)$ & \\
\hline $3 T$ & $198(30.4)$ & $136(30.2)$ & $62(30.7)$ & \\
\hline
\end{tabular}

ICA cav-cli-oph = cavernous and clinoid and ophthalmic segment of ICA; ICA com-bif = communicating segment to bifurcation of ICA; VBA = vertebral and basilar artery.

Values are presented as number of aneurysms (\%) unless otherwise indicated. Mean values are presented \pm SD.

specificity, and accuracy by location, size, and magnetic field strength (Tesla) of the MRA. If comparisons by location and size were statistically significant, multiple comparison results were performed using the Marascuilo procedure. Binary logistic regression was used to determine risk factors for false negatives with MRA of irregularly shaped aneurysms. In multivariate analysis, only cases in which false-negative findings were found by both reviewers 1 and 2 were used. The Cohen $\kappa$ statistic was used for comparison of observer performance for categorization of aneurysm shape. A p value of 0.05 was considered statistically significant.

\section{Results}

In total, 719 incidentally found intracranial aneurysms smaller than $7 \mathrm{~mm}$ were confirmed by DSA at our institution over the 2-year study period. We excluded 40 aneurysms that were first diagnosed with CTA, 5 aneurysms imaged with 0.5 T MRA, 20 aneurysms with a long interval between MRA and DSA (i.e., > 2 months), and 2 aneurysms with poor visualization due to artifacts. Finally, 652 unruptured intracranial aneurysms in 530 patients were evaluated morphologically for this study. By morphological subtype, 450 aneurysms had a regular shape and 202 aneurysms had an irregular shape according to DSA findings. By magnetic field strength, 454 aneurysms were acquired at 1.5T MRA and 198 aneurysms were obtained at 3T. The average size of irregularly shaped aneurysms was larger than that of regularly shaped aneurysms (3.28 $\pm 1.34 \mathrm{~mm}$ vs $4.66 \pm 1.22 \mathrm{~mm}, \mathrm{p}<0.001$ ). There was a difference in the ratio of irregularly shaped to regularly shaped aneurysms for each aneurysm location, and the proportion of irregularly shaped aneurysms was high in the ACA and MCA. Detailed baseline characteristics of patients and aneurysms are shown in Table 1.

The overall MRA sensitivity for detecting irregularity of aneurysm shape was $60.4 \%$ for reviewer 1 and $60.9 \%$ for reviewer 2 . The diagnostic performance parameters of MRA for aneurysms overall are detailed in Table 2.

Depending on the location of the aneurysm, MRA sensitivity for detecting the irregular shape of an ACA aneurysm was $36.7 \%$ for reviewer 1 and $46.9 \%$ for reviewer 2 , which was significantly lower than for other locations. The cavernous-clinoid-ophthalmic segment of ICA aneurysms had the highest sensitivity. The specificity was not signifi-

TABLE 2. Overall results for the aneurysms

\begin{tabular}{ccccccc}
\hline & AUC $(95 \%$ Cl) & Sensitivity (raw data) & Specificity (raw data) & PPV (raw data) & NPV (raw data) & Accuracy (raw data) \\
\hline Reviewer 1 & $86.1 \%(82.3-89.8)$ & $60.4 \%(122 / 202)$ & $98.4 \%(443 / 450)$ & $94.6 \%(122 / 129)$ & $84.7 \%(443 / 523)$ & $86.7 \%(565 / 652)$ \\
\hline Reviewer 2 & $85.0 \%(81.2-88.8)$ & $60.9 \%(123 / 202)$ & $97.6 \%(439 / 450)$ & $91.8 \%(123 / 134)$ & $84.7 \%(439 / 518)$ & $86.2 \%(562 / 652)$ \\
\hline
\end{tabular}


TABLE 3. Aneurysm location-based evaluations

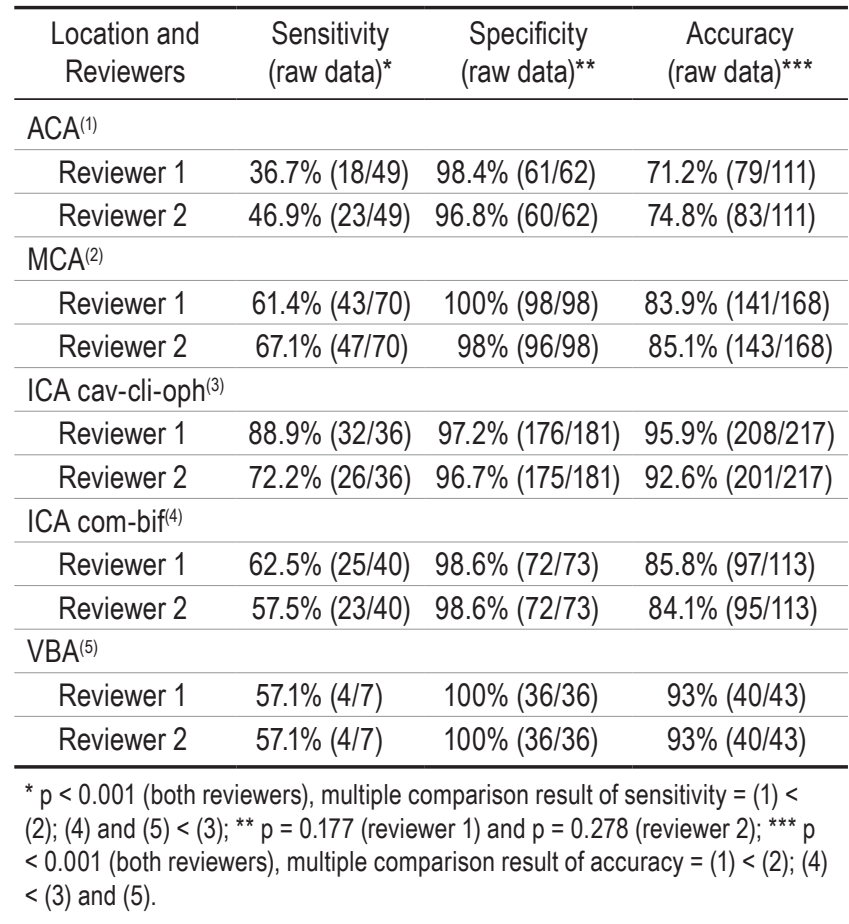

cantly different according to the location of the aneurysm. MRA performance parameters, depending on the location of the aneurysm, are detailed in Table 3.

The sensitivity of MRA for diagnosing irregularly shaped aneurysms was higher in the larger-sized aneurysm group (26.7\% [both reviewers], $<3 \mathrm{~mm} ; 54.5 \%$ [both reviewers], 3 to $4.9 \mathrm{~mm} ; 72.7 \%$ [reviewer 1] and $73.9 \%$ [reviewer 2], 5 to $6.9 \mathrm{~mm}$; $<<0.001$ ), while the MRA accuracy for $<3$-mm-sized aneurysms was significantly higher than the accuracy for the other aneurysm size groups. Specificity did not significantly differ for the size of the aneurysm. The detailed MRA performance values with regard to the size of the aneurysms are shown in Table 4 .

TABLE 4. Aneurysm size-based evaluations

\begin{tabular}{|c|c|c|c|}
\hline $\begin{array}{l}\text { Size and } \\
\text { Reviewers }\end{array}$ & $\begin{array}{l}\text { Sensitivity } \\
\text { (raw data) }^{*}\end{array}$ & $\begin{array}{l}\text { Specificity } \\
{\text { (raw data })^{* *}}\end{array}$ & $\begin{array}{c}\text { Accuracy } \\
(\text { raw data) })^{* * *}\end{array}$ \\
\hline \multicolumn{4}{|l|}{$<3 \mathrm{~mm}^{(1)}$} \\
\hline Reviewer 1 & $26.7 \%(4 / 15)$ & $99.1 \%(218 / 220)$ & $94.5 \%(222 / 235)$ \\
\hline Reviewer 2 & $26.7 \%(4 / 15)$ & $98.6 \%(217 / 220)$ & $94.0 \%(221 / 235)$ \\
\hline \multicolumn{4}{|l|}{$3-4.9 \mathrm{~mm}^{(2)}$} \\
\hline Reviewer 1 & $54.5 \%(54 / 99)$ & $98.2 \%(166 / 169)$ & $82.1 \%(110 / 134)$ \\
\hline Reviewer 2 & $54.5 \%(54 / 99)$ & $97.0 \%(164 / 169)$ & $81.3 \%(109 / 134)$ \\
\hline \multicolumn{4}{|l|}{$5-6.9 \mathrm{~mm}^{(3)}$} \\
\hline Reviewer 1 & $72.7 \%(64 / 88)$ & $96.7 \%(59 / 61)$ & $82.6 \%(123 / 149)$ \\
\hline Reviewer 2 & $73.9 \%(65 / 88)$ & $95.1 \%(58 / 61)$ & $82.6 \%(123 / 149)$ \\
\hline
\end{tabular}

TABLE 5. MRA magnetic field strength-based evaluations

\begin{tabular}{cccc}
\hline $\begin{array}{c}\text { Field Strength/ } \\
\text { Reviewers }\end{array}$ & $\begin{array}{c}\text { Sensitivity } \\
\text { (raw data) }\end{array}$ & $\begin{array}{c}\text { Specificity } \\
\text { (raw data) }\end{array}$ & $\begin{array}{c}\text { Accuracy } \\
\text { (raw data) }\end{array}$ \\
\hline 1.5T & & & \\
\hline Reviewer 1 & $52.9 \%(74 / 140)$ & $98.1 \%(308 / 314)$ & $84.1 \%(382 / 454)$ \\
\hline Reviewer 2 & $54.3 \%(76 / 140)$ & $97.1 \%(305 / 314)$ & $83.9 \%(381 / 454)$ \\
\hline 3T & & & \\
\hline Reviewer 1 & $77.4 \%(48 / 62)$ & $99.3 \%(135 / 136)$ & $92.4 \%(183 / 198)$ \\
\hline Reviewer 2 & $75.8 \%(47 / 62)$ & $98.5 \%(134 / 136)$ & $91.4 \%(181 / 198)$ \\
\hline
\end{tabular}

Depending on the magnetic strength of MRA, sensitivity and accuracy at $3 \mathrm{~T}$ were significantly better than at $1.5 \mathrm{~T}$. The specificity of the reviewer results for both reviewers did not reach statistical significance. Those results are detailed in Table 5.

Results of multivariate analysis of risk factors for falsenegative MRA findings in irregularly shaped aneurysms are shown in Table 6. Among the aneurysm locations, ACA had the highest odds of false negatives. Irregularly shaped ACA aneurysms were 13.03-fold more likely to be misjudged as smooth aneurysms than irregularly shaped cavernous-clinoid-ophthalmic segment ICA aneurysms (95\% CI 3.247-52.296; $\mathrm{p}<0.001$ ). Depending on the aneurysm size, the smaller-sized group of irregularly shaped aneurysms had higher odds of MRA false negatives. Aneurysms smaller than $3 \mathrm{~mm}$ with an irregular shape were associated with a significantly increased risk of 7.81-fold of misdiagnosis as regularly shaped aneurysms compared with irregularly shaped aneurysms $5-6.9 \mathrm{~mm}$ in size (95\% CI 2.12-28.80; $p=0.002)$. Lower MRA magnetic strength was also independently associated with increased odds of false negatives with irregularly shaped aneurysms (OR 4.54; 95\% CI 1.97-10.45; $\mathrm{p}<0.001$ ). The $\kappa$ value for overall aneurysm shape diagnosis was 0.665 , and $\kappa$ values

TABLE 6. Multivariate analysis for risk factors of MRA false negatives in irregularly shaped aneurysms

\begin{tabular}{cccc}
\hline & OR & $95 \% \mathrm{Cl}$ & p Value \\
\hline Location & & & \\
\hline ACA & 13.031 & $3.247-52.296$ & 0.000 \\
\hline MCA & 5.770 & $1.492-22.309$ & 0.011 \\
\hline ICA cav-cli-oph & 1.000 & & \\
\hline ICA com-bif & 6.396 & $1.530-26.742$ & 0.011 \\
\hline VBA & 5.658 & $0.666-48.087$ & 0.112 \\
\hline Size group & & & \\
\hline$<3$ mm & 7.816 & $2.121-28.803$ & 0.002 \\
\hline 3-4.9 mm & 3.341 & $1.557-7.171$ & 0.002 \\
\hline 5-6.9 mm & 1.000 & & \\
\hline MRA field strength & & & \\
\hline 1.5T & 4.539 & $1.971-10.449$ & 0.000 \\
\hline 3T & 1.000 & & \\
\hline
\end{tabular}

Results adjusted by age and sex. 
based on magnetic strength were 0.634 for 1.5T MRA and 0.725 for 3T MRA.

\section{Discussion}

We report an MRA sensitivity of $60 \%$ for detecting the irregular shape of small intracranial aneurysms. Several studies have reported a high sensitivity of MRA in the diagnosis of intracranial aneurysms. In a meta-analysis, the sensitivity of MRA in detecting aneurysms was $95 \%$. Our findings demonstrate that, unlike detection of the presence of an aneurysm, MRA is not appropriate for determining the shape of an aneurysm for small aneurysms. Irregular shape is one of the risk factors for rupture of unruptured intracranial aneurysms, irrespective of size ${ }^{6,8}$ In our study, around $40 \%$ of irregularly shaped aneurysms with a high risk of rupture were misjudged as low-risk smooth aneurysms.

Findings of some previously reported studies, unlike our findings, have demonstrated that MRA is also highly accurate in identifying aneurysm shapes. ${ }^{2,3}$ However, these studies were performed under the conditions of a strict academic setting using identical MRA protocols and specialized postprocessing techniques, conditions that do not necessarily apply in an actual clinical setting. In clinical practice, most incidental intracranial aneurysm patients undergo MRA at institutions other than those at which they undergo aneurysm treatment. Thus, clinical variability in MRA technology and postprocessing techniques is an unavoidable limitation of studies performed in patients treated at various institutions. In another study, conducted in an actual clinical setting, a 59\% inaccuracy rate was reported for the diagnosis of intracranial aneurysms. ${ }^{9}$ In addition, the aforementioned studies detecting aneurysm shape achieved high accuracy and sensitivity using volume-rendered MRA images. Volume-rendered images allow better visualization of the contour of an overlaid area of an artery or aneurysm and thus definitely help identify the shape of an aneurysm. However, volume-rendering reconstruction requires a specialized software protocol, takes more time to process, and has not yet been widely used. Furthermore, an aneurysm is often found in a patient who has undergone MRA for other reasons, for example during stroke evaluation. In such cases, volume-rendered MRA is rarely used. Indeed, most MRAs evaluated in our study did not include volume-rendered images.

\section{Aneurysm Location}

False-negative findings of irregularly shaped aneurysms identified in our study were most common in the ACA region. According to our results, it is likely that MRA often fails to detect a small bleb or lobulation of an aneurysm, especially in the ACA, because it is assumed that overlapping areas of 2 ACAs and an aneurysm are large. In addition, flow artifacts in the anterior communicating artery may cause poor visualization of aneurysm shape in this region. ${ }^{10}$ False-positive irregularly shaped aneurysms were identified most commonly in the region of the cavernous-clinoid-ophthalmic segment of the ICA. In the cavernous and clinoidal regions of the ICA, artifactual signal degradation occurs routinely ${ }^{11}$ and the air/bone structure at this region, such as the pneumatized anterior clinoid process or sphenoid sinus, can generate artifacts. ${ }^{12}$ Such artifacts may distort the shape of an aneurysm with a smooth surface.

\section{Aneurysm Size}

In our study, MRA was highly accurate for aneurysms smaller than $3 \mathrm{~mm}$, but the sensitivity was significantly low. The reason for this result may be that in our study there was a predominance of regularly shaped aneurysms $<3 \mathrm{~mm}$ (15 irregular vs 220 regular) and irregular shape was more common when the size of the aneurysm was large. Although the accuracy was the highest because of the high rate of true negatives, MRA mostly failed to detect irregular shape in aneurysms $<3 \mathrm{~mm}$. The smaller the aneurysm size, the less likely the irregular shape was to be identified by MRA. Li et al. ${ }^{13}$ reported that the sensitivity of MRA was $98.2 \%-98.7 \%$ for the diagnosis of small ( $\leq$ $5 \mathrm{~mm}$ ) intracranial aneurysms. However, for detection of irregular aneurysm shape in our study, the MRA sensitivity was only $50.9 \%$ (58/114) with aneurysms $<5 \mathrm{~mm}$ in diameter.

\section{MRA Field Strength (1.5T vs $3 \mathrm{~T}$ )}

Not surprisingly, 3T MRA showed better diagnostic performance than 1.5T MRA in identifying aneurysm shape in our study. Imaging at 3T provides superior spatial resolution, improved vessel contrast, and better background tissue and fat suppression, which provide superior image quality and better visualization of intracranial vessels..$^{14,15}$ Until recently, however, many institutions still used a 1.5T MRI scanner. In fact, our study confirmed that in our local medical community there were more than twice as many $1.5 \mathrm{~T}$ than 3T MRA images obtained. Therefore, at an actual outpatient clinic, clinicians who make choices regarding therapeutic decisions may not have 3T MRA as an option.

\section{CT Angiography}

CTA could be an alternative to MRA or DSA. CTA has a shorter imaging time than MRA and is less invasive than DSA. Several studies have reported high diagnostic accuracy with CTA. For aneurysms smaller than $5 \mathrm{~mm},{ }^{16,17}$ many studies have reported that CTA has a higher sensitivity than MRA and even DSA, and several studies have suggested that CTA may one day replace DSA. ${ }^{18-21}$ However, previous studies have shown mixed results in detecting aneurysmal shape with CTA. ${ }^{3,20,22}$ The agreement ratio of DSA versus CTA regarding aneurysm shape was $96 \%$ in one study, ${ }^{3}$ but another study showed a Cohen $\kappa$ coefficient for CTA of only 0.21 between two reviewers when determining cerebral aneurysm shape. ${ }^{22}$ Inaccuracies in determining aneurysmal shape with CTA were suggested to result from the mismatch between scan timing and contrast enhancement inside the aneurysm or confusion with parts of bony structures adjacent to aneurysms. ${ }^{22} \mathrm{In}$ addition, CTA does not appear to be more widely used than DSA for diagnosing intracranial aneurysms. Patients with an unruptured intracranial aneurysm who underwent DSA were rarely first diagnosed with CTA at our outpa- 
tient clinic. We suspect that physicians may be reluctant to obtain CTA images due to concerns about radiation risk with CTA and its high load of contrast agent. The iodinated contrast agent used for CTA can be nephrotoxic, and the volume injected for CTA is typically higher than the amount of contrast used for routine DSA.

\section{Study Limitations}

There are a few limitations to this study. First, because this was a retrospective single-center study, there may be selection bias. However, since the study has a large cohort and the patients were consecutive, selection bias may have been minimized. Second, different MRI scanners were used at different facilities for this study. This may have caused differences in interpreting the imaging findings. Specific types of MR equipment or specific algorithms may be more effective in showing the shape of aneurysms. However, since most of the MRAs were taken at different institutions, it was difficult to confirm all of the details of the algorithms used in imaging. Moreover, because of the wide variety of MR scanner types in this study, we were unable to compare them statistically. Third, aneurysm development may have occurred during the interval between MRA and DSA. Although we excluded patients with an interval longer than 2 months to reduce the number of aneurysms that changed in size over the course of our study, it is a limitation of our study that the two procedures were implemented on separate days. Therefore, a prospective, multicenter study to evaluate the accuracy of MRA in identifying the shape of intracranial aneurysms will be required for accurate decision making for patients with small unruptured intracranial aneurysms.

\section{Conclusions}

Based on the results of this study, the sensitivity of MRA for detecting the irregular shape of small intracranial aneurysms was low. In particular, the sensitivity was significantly lower with ACA aneurysms and aneurysms $<3 \mathrm{~mm}$ in diameter, and with 1.5T MRA. Therefore, we suggest that DSA is needed for decision making in patient treatment, because MRA findings alone are not sufficient to determine the shape of small unruptured intracranial aneurysms.

\section{Acknowledgments}

This work was supported by a research grant from Daegu Catholic University in 2018 (grant no. 20183014).

\section{References}

1. Atlas SW, Sheppard L, Goldberg HI, et al. Intracranial aneurysms: detection and characterization with MR angiography with use of an advanced postprocessing technique in a blinded-reader study. Radiology. 1997;203(3):807-814.

2. Cirillo M, Scomazzoni F, Cirillo L, et al. Comparison of 3D TOF-MRA and 3D CE-MRA at 3T for imaging of intracranial aneurysms. Eur J Radiol. 2013;82(12):e853-e859.

3. Hiratsuka Y, Miki H, Kiriyama I, et al. Diagnosis of unruptured intracranial aneurysms: 3T MR angiography versus 64-channel multi-detector row CT angiography. Magn Reson Med Sci. 2008;7(4):169-178.
4. Mallouhi A, Felber S, Chemelli A, et al. Detection and characterization of intracranial aneurysms with MR angiography: comparison of volume-rendering and maximum-intensityprojection algorithms. AJR Am J Roentgenol. 2003;180(1): 55-64.

5. Hademenos GJ, Massoud TF, Turjman F, Sayre JW. Anatomical and morphological factors correlating with rupture of intracranial aneurysms in patients referred for endovascular treatment. Neuroradiology. 1998;40(11):755-760.

6. Lindgren AE, Koivisto T, Björkman J, et al. Irregular shape of intracranial aneurysm indicates rupture risk irrespective of size in a population-based cohort. Stroke. 2016;47(5): 1219-1226.

7. Sailer AM, Wagemans BA, Nelemans PJ, et al. Diagnosing intracranial aneurysms with MR angiography: systematic review and meta-analysis. Stroke. 2014;45(1):119-126.

8. Kleinloog R, de Mul N, Verweij BH, et al. Risk factors for intracranial aneurysm rupture: a systematic review. Neurosurgery. 2018;82(4):431-440.

9. Schwab KE, Gailloud P, Wyse G, Tamargo RJ. Limitations of magnetic resonance imaging and magnetic resonance angiography in the diagnosis of intracranial aneurysms. Neurosurgery. 2008;63(1):29-35.

10. Takhtani D, Dundamadappa S, Puri AS, Wakhloo A. Flow artifact in the anterior communicating artery resembling aneurysm on the time of flight MR angiogram. Acta Radiol. 2014;55(10):1253-1257.

11. Korogi Y, Takahashi M, Mabuchi N, et al. Intracranial aneurysms: diagnostic accuracy of three-dimensional, Fourier transform, time-of-flight MR angiography. Radiology. 1994; 193(1):181-186.

12. Deutschmann HA, Augustin M, Simbrunner J, et al. Diagnostic accuracy of 3D time-of-flight MR angiography compared with digital subtraction angiography for follow-up of coiled intracranial aneurysms: influence of aneurysm size. AJNR Am J Neuroradiol. 2007;28(4):628-634.

13. Li MH, Li YD, Gu BX, et al. Accurate diagnosis of small cerebral aneurysms $\leq 5 \mathrm{~mm}$ in diameter with 3.0-T MR angiography. Radiology. 2014;271(2):553-560.

14. Al-Kwifi O, Emery DJ, Wilman AH. Vessel contrast at three Tesla in time-of-flight magnetic resonance angiography of the intracranial and carotid arteries. Magn Reson Imaging. 2002; 20(2):181-187.

15. Gibbs GF, Huston J III, Bernstein MA, et al. Improved image quality of intracranial aneurysms: $3.0-\mathrm{T}$ versus $1.5-\mathrm{T}$ time-offlight MR angiography. AJNR Am J Neuroradiol. 2004;25(1): $84-87$.

16. Tanabe S, Ohtaki M, Uede T, et al. Diagnosis of ruptured and unruptured cerebral aneurysms with three-dimensional CT angiography (3D-CTA). Article in Japanese. No Shinkei Geka. 1995;23(9):787-795.

17. Villablanca JP, Jahan R, Hooshi P, et al. Detection and characterization of very small cerebral aneurysms by using 2D and 3D helical CT angiography. AJNR Am J Neuroradiol. 2002;23(7):1187-1198.

18. Hoh BL, Cheung AC, Rabinov JD, et al. Results of a prospective protocol of computed tomographic angiography in place of catheter angiography as the only diagnostic and pretreatment planning study for cerebral aneurysms by a combined neurovascular team. Neurosurgery. 2004;54(6):1329-1342.

19. Kouskouras $C$, Charitanti A, Giavroglou C, et al. Intracranial aneurysms: evaluation using CTA and MRA. Correlation with DSA and intraoperative findings. Neuroradiology. 2004; 46(10):842-850.

20. Li Q, Lv F, Yao G, et al. 64-section multidetector CT angiography for evaluation of intracranial aneurysms: comparison with 3D rotational angiography. Acta Radiol. 2014;55(7): 840-846.

21. Pechlivanis I, Schmieder K, Scholz M, et al. 3-Dimensional 
computed tomographic angiography for use of surgery planning in patients with intracranial aneurysms. Acta Neurochir (Wien). 2005;147(10):1045-1053.

22. Franklin B, Gasco J, Uribe T, et al. Diagnostic accuracy and inter-rater reliability of 64-multislice 3D-CTA compared to intra-arterial DSA for intracranial aneurysms. J Clin Neurosci. 2010;17(5):579-583.

\section{Disclosures}

The authors report no conflict of interest concerning the materials or methods used in this study or the findings specified in this paper.

\section{Author Contributions}

Conception and design: Kang, Park. Acquisition of data: Kwak, Son. Analysis and interpretation of data: Kang, Kim. Drafting the article: Kwak. Statistical analysis: Kwak. Administrative/techni$\mathrm{cal} /$ material support: Son, Park. Study supervision: Kang.

\section{Supplemental Information \\ Current Affiliations}

Dr. Kwak: Department of Neurosurgery, School of Medicine, Kyungpook National University, Daegu, Republic of Korea.

\section{Correspondence}

Dong-Hun Kang: Kyungpook National University Hospital, Daegu, Republic of Korea.kdhdock@gmail.com. 